\title{
Magnetic Exchange Bias and Its Ion Beam Induced Enhancement in a Nanoscale Si/Co/Si System
}

\author{
Sumalay Roy ${ }^{1}$, Miklós Menyhárd ${ }^{2}$, Subham Majumdar ${ }^{3}$, Dmitri Novikov ${ }^{4}$ and Bhupendra Nath Dev $^{1}$ \\ 1. Department of Materials Science, Indian Association for the Cultivation of Science, Kolkata 700032, India \\ 2. Research Institute for Technical Physics and Materials Science, Budapest 1525, Hungary \\ 3. Department of Solid State Physics, Indian Association for the Cultivation of Science, Kolkata 700032, India \\ 4. Hamburg Synchrotron Radiation Laboratory, HASYLAB at DESY, Notkrestasse 85, Hamburg D-22607, Germany
}

Received: June 15, 2010 / Accepted: July 09, 2010 / Published: February 10, 2011.

\begin{abstract}
Measurements of magnetic moment (M) as a function of applied magnetic field $(\mathrm{H})$ have been carried out using a superconducting quantum interference device (SQUID) on an ultrathin bilayer film Si/Co, deposited on a silicon substrate. The M-H hysteresis loop has shown a shift with respect to the $\mathrm{H}=0$ axis due to the presence of a unidirectional or exchange anisotropy in the material. The amount of shift of the loop along the $\mathrm{H}$ axis, which is known as the exchange bias field $H_{E}$ has been measured for the as-grown as well as the ion irradiated samples. Ion irradiation was carried out with $2 \mathrm{MeV} \mathrm{Cu}^{++}$ions at different fluences ranging from $1 \times 10^{13}$ ions $/ \mathrm{cm}^{2}$ to $1 \times 10^{15}$ ions $/ \mathrm{cm}^{2}$. The value of $H_{E}$ has been found to increase with increasing ion fluence. X-ray reflectivity (XRR) measurements on the as-grown as well as the ion irradiated samples show an increase in mixing of $\mathrm{Si}$ and $\mathrm{Co}$ at the $\mathrm{Co} / \mathrm{Si}$ interfaces with the increase in fluence. Depth profile of different elements by Auger electron spectroscopy (AES) also supports this strong Si-Co mixing at the $\mathrm{Si} / \mathrm{Co}$ interfaces. Ion beam induced enhancement of exchange bias has been attributed to a reduction of the thickness of the Co layer due to alloying at the interfaces as well as a reduction in the saturation magnetization due to incorporation of some $\mathrm{Si}$ into the Co layer. The interface exchange coupling energy has also been estimated for this system.
\end{abstract}

Key words: Thin film, magnetic exchange bias, X-ray reflectivity, ion irradiation induced modifications, Co-Si alloy.

\section{Introduction}

Interaction of energetic ion beams with artificially structured solid materials can give rise to interesting and useful phenomena. Utilizing such phenomena ion beams have been used to fabricate modified materials for diverse electronic, optical and tribological applications. In recent years ion beam induced phenomena regarding magnetic behavior have gained importance. Ion irradiation of magnetic multilayers, e.g. $\mathrm{Co} / \mathrm{Pt}$ multilayers, has been found to show a spin-orientation transition, indicating the suitability of such systems for patterned ultrahigh density magnetic

Corresponding author: Bhupendra Nath Dev (1953-), male, senior professor, research fields: surface and nanoscale science, ion-solid interactions and X-ray physics. E-mail: msbnd@iacs.res.in. recording media [1]. Spin orientation transition or loss of perpendicular anisotropy has been attributed to ion beam induced interfacial intermixing between Co and Pt. Ion beam induced atomic migration across interfaces in a multilayer structure [2] is capable of transforming a nonmagnetic system into a ferromagnetic system and the variation of ion-fluence can tune the coercive field [3]. Structural analysis has shown that ion beam irradiation causes various micro-structural modifications, e.g., intermixing, interface broadening and change in layer thickness [4]. While intermixing at the interface was thought to be responsible for spin-orientation transition, other aspects, e.g. change of layer thickness, may also be responsible for modification of magnetic behavior, the most spectacular example of which are multilayers of 
alternating magnetic and nonmagnetic layers. These multilayers show giant magnetoresistance (GMR) where the magnetic coupling switches between ferromagnetic and antiferromagnetic depending on the thickness of the nonmagnetic layer [5, 6]. In magnetic multilayers, where alternating layers are magnetic and nonmagnetic materials, magnetic coupling and magnetoresistance can be changed by introducing some magnetic impurity atoms in the non-magnetic layers and controlling their concentrations [7]. This can be done by ion irradiation as ion beam induced displaced magnetic atoms can be incorporated into the neighboring nonmagnetic layers and their concentration can be determined with high precision [4]. Ion irradiation can even induce ferromagnetism in unconventional materials like fullerene and other carbon materials [8]. The topic of tailoring magnetism by light-ion irradiation has been recently reviewed by Fassbender et al. [9]. In the present work, we address the issue of exchange bias and its ion beam induced enhancement in a $\mathrm{Si} / \mathrm{Co} / \mathrm{Si}$ trilayer system.

The magnetic anisotropy which manifests itself in a shift in the magnetic hysteresis loop (typically the M-H loop in the magnetic moment $(\mathrm{M})$ versus applied magnetic field ( $\mathrm{H}$ ) curve) with respect to the $\mathrm{H}=0$ axis, is known as unidirectional or exchange anisotropy for the material under consideration and is still a subject matter of intense scientific research though it was discovered more than 50 years ago [10]. This phenomenon of hysteresis loop shift is usually observed in a material where at least two material components of different magnetic properties, e.g., ferromagnets, antiferromagnets, ferrimagnets and spin glasses are combined and have interfacial coupling. It was observed for the first time in a core-shell structure, where a ferromagnetic (FM) core (Co) magnetically coupled with an antiferromagnetic (AFM) shell (CoO) gives rise to a shift in the hysteresis loop $[10,11]$. Basically, when a sample with an FM-AFM interface is cooled in a static magnetic field $(\mathrm{H})$ from above the Neel temperature $\left(\mathrm{T}_{N}\right)$ of the AFM material, and the
FM Curie temperature is greater than $\mathrm{T}_{N}$, the magnetic hysteresis loop shifts from the $\mathrm{H}=0$ axis. The magnitude of this shift is known as exchange bias (EB) field and denoted by $H_{E}$. As of now, the predominant material systems, besides these core-shell like structures, which exhibit exchange anisotropy are: inhomogeneous spin glasses where magnetic coupling between FM and AFM domains take place [12], thin films (of a few nanometer thicknesses) consisting of bilayers where a FM layer couples magnetically to an AFM layer [13], or a system of double superlattice where an artificial FM superlattice interacts with an AFM superlattice [14], or bilayer thin films which magnetically combine a FM layer with a ferrimagnetic layer [15]. Although some research work has been carried out with nanostructured core-shell systems, in the last decades, almost the entire research on EB was focused mainly on FM-AFM thin film systems due to the possibility of an increased number of FM-AFM combinations in thin films and more importantly, the fundamental role of EB in spin valve and tunneling devices [16]. In these FM-AFM bilayer thin films the AFM layer acts as a 'pinning' layer which 'pins' the spins of the FM layer near the interface and gives rise to the EB effect [16]. Though it is known that an interface spin structure plays an important role in the EB effect the proper understanding is still lacking. The technological applications together with the fact that the proper understanding of the underlying mechanism of EB phenomena is not well-understood yet, thin film FM-AFM structure remains a very important system for research till date [16].

Amorphous and nanocrystalline metal-metalloid thin films have been a very interesting subject matter of research due to the possibility to study the interplay of their magnetic properties, crystallographic structure and microstructural features. Many studies have already been carried out on these aspects, but most of them are centered around $\mathrm{Fe}-\mathrm{Si}$ based alloy thin films $[17,18]$ while considerably little effort was dedicated towards understanding of the corresponding Co based 
materials [19, 20]. Currently multilayers, which are made of alternating layers of a ferromagnetic and a non-magnetic semiconductor materials and potential candidate for development of magnetic random access memory, are finding great attention due to their very controversial nature and inadequate theoretical understanding. The $\mathrm{Fe} / \mathrm{Si}$ multilayer system has been extensively studied and a correlation between their magnetic properties and microstructural details has been found [21-24]. In comparison to the $\mathrm{Fe} / \mathrm{Si}$ multilayer system the $\mathrm{Co} / \mathrm{Si}$ multilayer system is less studied despite its higher technological importance. Observations on $\mathrm{Co} / \mathrm{Si}$ multilayers by Fallon and his co-workers $[25,26]$ reveal the importance of formation of Co-Si compounds and alloys due to the mixing of Co and $\mathrm{Si}$ at the interfaces. In a recent work AFM coupling has been observed in an amorphous $\mathrm{Co}_{x} \mathrm{Si}_{1-\mathrm{x}} / \mathrm{Si}$ multilayer and magnetic properties of thin films of $\mathrm{Co}_{x} \mathrm{Si}_{1-\mathrm{x}}$ deposited on Si were also studied [27].

Along with other modifications in magnetic properties in nanoscale thin films and multilayers, as discussed earlier, the use of ion beam irradiation has also led to important effects on a system which shows exchange bias properties. Light ion irradiation can be used to tune the exchange bias $\left(H_{E}\right)$ and the coercivity $\left(H_{C}\right)$ of FM-AFM bilayers $[28,29]$. Very recently, it has been observed that swift heavy ion irradiation on a $\mathrm{Fe} / \mathrm{Si}$ bilayer system has produced a very small exchange bias, which was otherwise absent in the as-deposited system [30].

In this work, we have taken up a $\mathrm{Si} / \mathrm{Co} / \mathrm{Si}$ trilayer system, where a Co layer and a Si layer were deposited sequentially on a $\mathrm{Si}$ (111) wafer substrate under ultrahigh vacuum (UHV) condition. Small pieces were cut from this wafer. These samples were irradiated with accelerated heavy ions at different fluences. Hysteresis loop (M-H) measurements using superconducting quantum interference device (SQUID) magnetometry have shown the presence of $H_{E}$ in the as-grown sample and a gradual increase of $H_{E}$ with increase in ion fluence in the irradiated samples even at room temperature (RT). Dependence of exchange bias on the ion fluence in irradiation has been analyzed. For an understanding of the evolution of magnetic behavior, microstructural study using x-ray reflectivity (XRR) and depth profiling by Auger electron spectroscopy (AES) were carried out. Both these analyses indicate a strong mixing between $\mathrm{Co}$ and $\mathrm{Si}$ at the interfaces to form a $\mathrm{Co}_{\mathrm{x}} \mathrm{Si}_{1-\mathrm{x}}$ alloy at the $\mathrm{Co} / \mathrm{Si}$ interfaces and a reduction of the Co layer thickness. The observed changes in the magnetic behavior due to ion irradiation are explained in terms of the presence of $\mathrm{Co} / \mathrm{Co}_{\mathrm{x}} \mathrm{Si}_{1-\mathrm{x}}$ interfaces and the thickness modification of the Co layer.

\section{Experimental Details}

$\mathrm{Si}$ (111) surfaces were produced under ultrahigh vacuum (UHV) condition in a molecular beam epitaxy system. A Si buffer layer was first grown on the $\mathrm{Si}(111)$ substrate $(100 \mathrm{~mm}$ diameter $)$ at an elevated temperature $\left(700{ }^{\circ} \mathrm{C}\right)$. A Co layer $(\sim 10 \mathrm{~nm})$ was deposited on the buffer layer from an electron beam source with the substrate at room temperature. A thin $(\sim 5 \mathrm{~nm})$ amorphous $\mathrm{Si}$ layer was then deposited on the Co layer and the sample was taken out of the UHV environment. A part of this as-grown sample was cut into two series ( $\mathrm{L}$ and $\mathrm{S}$ ) of several pieces. The sample dimensions for L (large) and S (small) series are $(7 \times 14)$ $\mathrm{mm}^{2}$ and $(4 \times 6) \mathrm{mm}^{2}$ respectively. The small samples ( $\mathrm{S}$ series) are for SQUID measurements and the large samples (L series) are for XRR measurements. A 2 $\mathrm{MeV} \mathrm{Cu}{ }^{++}$ion beam with three different fluences $\left(3 \times 10^{14}\right.$ ions $/ \mathrm{cm}^{2}, 6 \times 10^{14}$ ions $/ \mathrm{cm}^{2}$ and $1 \times 10^{15}$ ions $\left./ \mathrm{cm}^{2}\right)$ was rastered on three different sets of $\mathrm{L}$ and $\mathrm{S}$. In each case one $\mathrm{L}$ and one $\mathrm{S}$ sample were irradiated simultaneously, using the $3 \mathrm{MV}$ tandem Pelletron accelerator at Institute of Physics, Bhubaneswar, India. The choice of beam energy and non-magnetic ion species was made such that the ions penetrate deep into the substrate and do not interact magnetically with the Co layer. Hysteresis loop (M-H) loop measurement on the S-series samples (as-grown as well as ion beam 
irradiated) was made using our in-house SQUID system. All M-H data reported here were obtained at room temperature (RT). The L (large) series samples were used in X-ray reflectivity (XRR) measurements. The XRR experiments were carried out at the ROMÖ-I beamline at Hamburg Synchrotron Radiation Laboratory (HASYLAB), DESY, Germany using 14 $\mathrm{keV}$ monochromatized X-rays. The incident monochromatic beam was obtained using a $\mathrm{Si}(111)$ double crystal monochromator. The reflected intensity was measured using a scintillation (NaI) detector. The S-series samples were again cut into smaller pieces and AES depth profiling was carried out on them at Research Institute for Technical Physics and Materials Science at Budapest, Hungary. Depth profiling was carried out by sputtering the samples using a $0.5 \mathrm{keV}$ $\mathrm{Ar}^{+}$beam, which was incident on the sample at $75^{\circ}$ with respect to the surface normal and atomic concentrations at different depths were found out from the analysis of AES at the corresponding depths.

\section{Results and Discussion}

The result of measurement of magnetic moment as a function of applied magnetic field at RT for the as-grown sample is shown in Fig. 1. The measured M-H loop shows a loop shift, i.e., exchange bias towards the positive side of the $\mathrm{H}$ axis. The exchange bias, $H_{E}$, is defined as (Fig. 1),

$$
H_{E}=\left(H_{C 1}+H_{C 2}\right) / 2
$$

Fig. 2a shows M-H loops measured for the as-grown as well as the ion beam irradiated samples at different fluences. Fig. 2a clearly shows that the M-H loops shift more and more away from the $\mathrm{H}=0$ axis as the ion beam fluence is increased progressively from zero (i.e. the as-grown sample) to $1 \times 10^{15}$ ions $/ \mathrm{cm}^{2}$. The $H_{E}$ values are extracted from all the $\mathrm{M}-\mathrm{H}$ loops and plotted (filled squares) as a function of ion-beam fluence in Fig. $2 \mathrm{~b}$. The values represented by open circles in Fig. $2 \mathrm{~b}$ will be discussed later. The coercive fields $\left(H_{C}\right)$ for all the samples are also obtained from the $\mathrm{M}-\mathrm{H}$ loops using the relation,

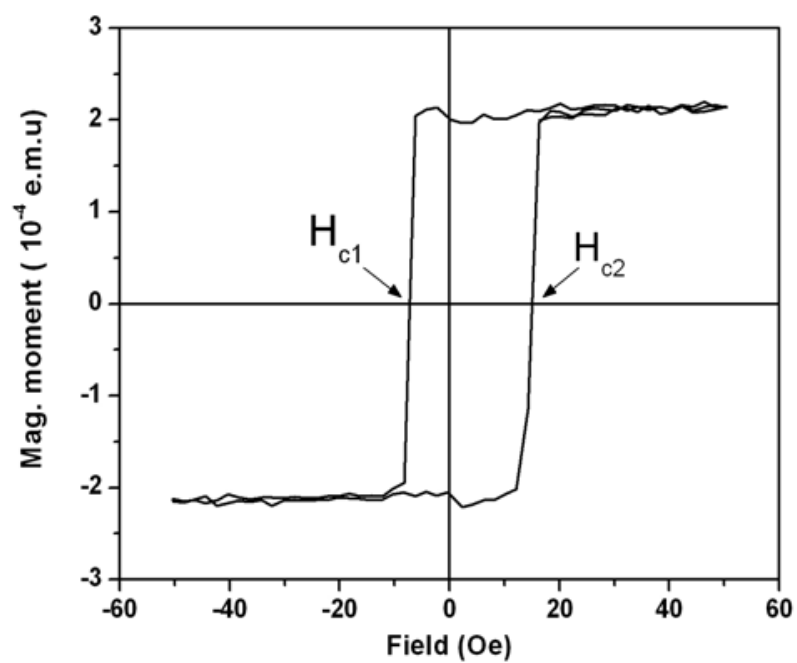

Fig. 1 Measured hysteresis loops (M-H loops) from the as-grown sample. Sample orientation with respect to the applied field is shown in top left corner. The easy axis is along the length of the sample.

$$
H_{C}=\left|\left(H_{C 1}-H_{C 2}\right)\right| / 2
$$

The value of $H_{C}$ is 9.1 Oe for the as-deposited sample and it increases with ion-fluence to $13.1 \mathrm{Oe}$ for the sample irradiated at the highest fluence $\left(1 \times 10^{15}\right.$ ions $/ \mathrm{cm}^{2}$ ). The saturation magnetic moment reduces with increase in ion fluence because of enhancement of mixing between $\mathrm{Co}$ and $\mathrm{Si}$ at the interfaces and consequent reduction in the thickness of the Co layer as well as somewhat reduction in Co concentration due to some $\mathrm{Si}$ incorporation in the Co layer. A close inspection of the $\mathrm{M}-\mathrm{H}$ loops show a positive value of magnetic moment even at $\mathrm{H}=0$ for all the curves. This implies that the ferromagnetic domains were previously aligned in a preferred direction. Presence of a very small magnetic field during the growth of the sample could give rise to an initial magnetization. However the question arises: what is the origin of an exchange bias in the system?

\subsection{Microstructure and Its Ion Beam Induced Evolution}

Since $\mathrm{CoO}$ is an AFM material which can couple to the FM Co layer to give rise to an exchange bias, in our thin film system, it is important to investigate if there has been oxygen diffusion into the system and consequent formation of $\mathrm{CoO}$ at the interfaces. We have carried out a depth profiling of various species by 

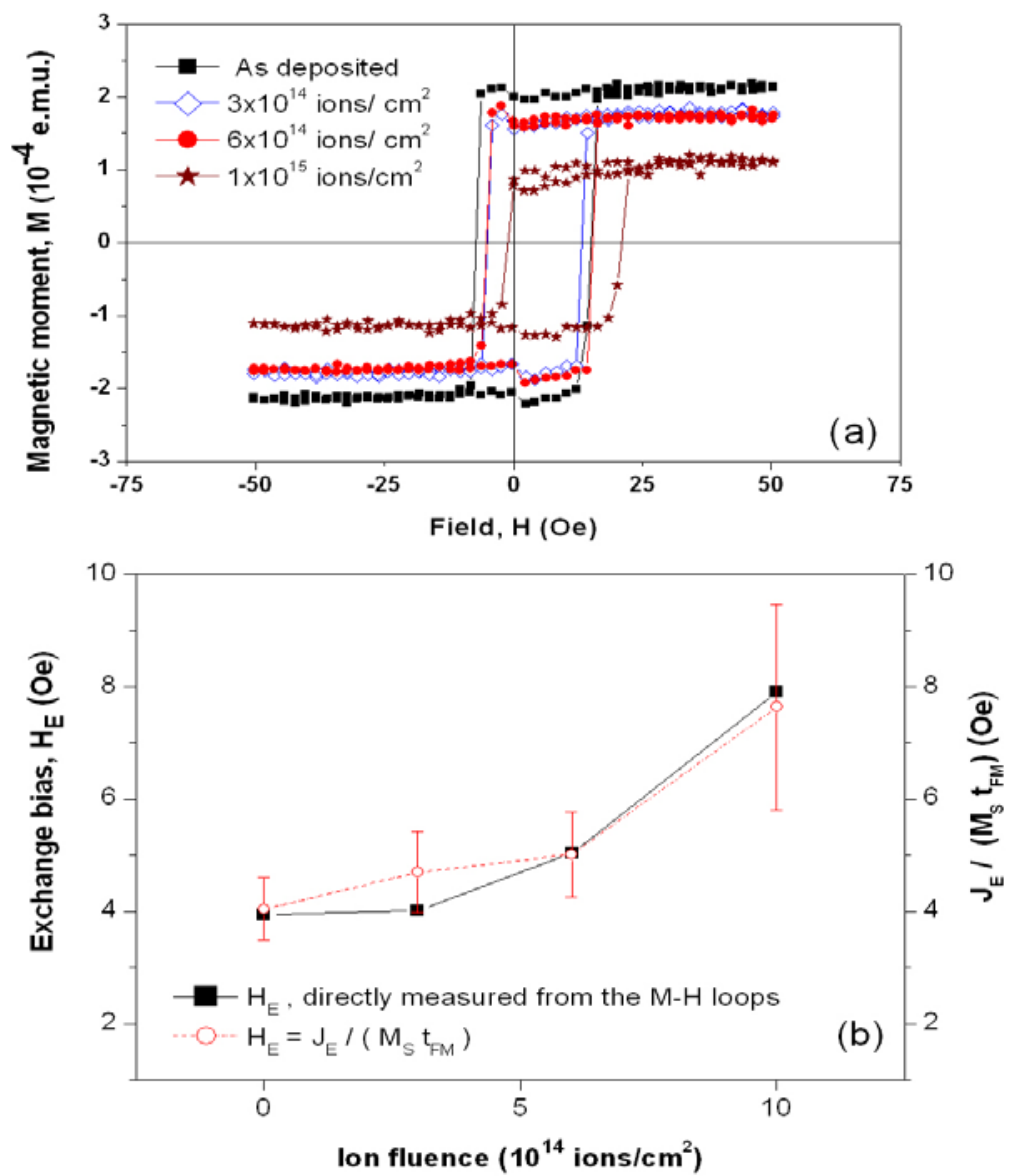

Fig. 2 (a) Measured M-H loops for as-grown as well as ion beam irradiated samples. (b) The values of $H_{E}$ extracted from each M-H loops in Fig. 2a has been plotted against the respective fluence. $H_{E}$ values estimated from Eq. (1) are also shown.

AES to find out any possible presence of $\mathrm{CoO}$ in contact with $\mathrm{Co}$ at the top $\mathrm{Si} / \mathrm{Co}$ and the bottom $\mathrm{Co} / \mathrm{Si}$ interfaces. The results are shown in Fig. 3. No significant presence of oxygen was detected either in the thin film or at the interfaces. So the possibility of formation of AFM CoO in contact with the Co layer at the interfaces was ruled out. However, all the depth profiles including the one for the as-deposited sample indicate strong mixing of $\mathrm{Co}$ and $\mathrm{Si}$ at both the $\mathrm{Co} / \mathrm{Si}$ interfaces. It is also apparent from the profiles that there is an increasing Co-Si mixing at the interfaces with increase in ion fluence. This $\mathrm{Co}-\mathrm{Si}$ mixing produces $\mathrm{Co}_{\mathrm{x}} \mathrm{Si}_{1-\mathrm{x}}$ alloys at the interfaces, which in turn reduces the effective layer thickness of FM Co with increasing ion-fluence. Also we notice the presence of a very thin layer of $\mathrm{C}$ on the surface of the ion irradiated samples. This is usual for ion irradiation under high vacuum $\left(\sim 10^{-6} \mathrm{mbar}\right)$ condition. At this pressure in the irradiation chamber there is a considerable presence of hydrocarbons. The cracking of hydrocarbons by the ion beam in front of the sample is the cause of $\mathrm{C}$ deposition. This $\mathrm{C}$ layer, in no way, is expected to affect the magnetic behavior. We also notice the presence of some $\mathrm{SiO}_{2}$ due to surface oxidation of the top Si layer.

Fig. 4 shows experimental XRR data and their theoretical simulations for as-grown as well as irradiated samples. X-ray reflectivity is a very powerful technique to measure microstructural details (e.g. surface and interface roughnesses, electron densities, layer thicknesses etc.) for nanoscale thin films and layered structures and has been widely discussed over the years [31-33]. The microstructural details come as parameters for fitting a theoretical curve to the 

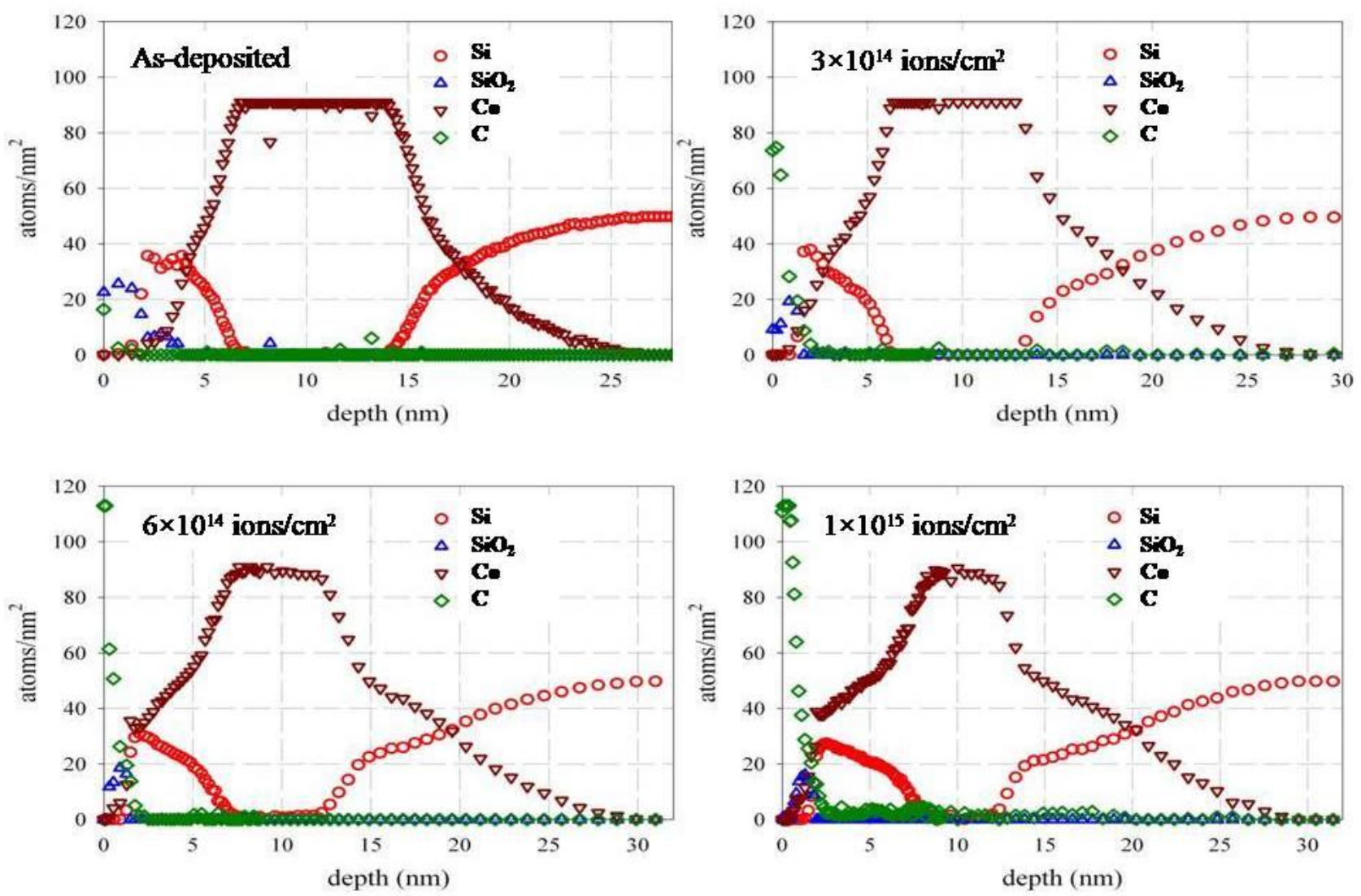

Fig. 3 AES depth profiles of different elements (and $\left.\mathrm{SiO}_{2}\right)$ and their areal density at different depths.

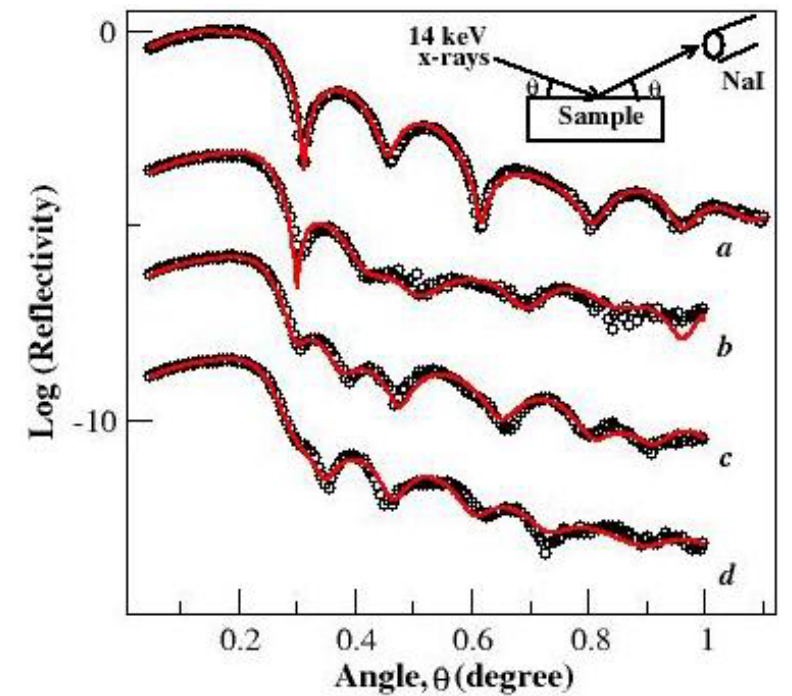

Fig. 4 Experimental $X$-ray reflectivity data (circles) and the fitted theoretical reflectivity curves (solid lines) for the as-deposited (a) and the ion-irradiated samples $(b, c$ and $d$ for $3 \times 10^{14}$ ions $/ \mathrm{cm}^{2}, 6 \times 10^{14}$ ions $/ \mathrm{cm}^{2}, 1 \times 10^{15}$ ions $/ \mathrm{cm}^{2}$ ion-fluences respectively). Data for the samples, except the as-deposited one, are vertically shifted down for clarity. The inset at top-right corner shows the experimental geometry. experimental observations. Here we use a multilayer-model dependent approach $[34,35]$ to fit the theoretically generated curve to the experimental XRR data. We took help from the AES depth profile to build up the theoretical model. The multilayer model used here to simulate the theoretical curves for fitting to the experimental data is shown in Fig. 5. We have considered two additional layers of $\mathrm{Co}-\mathrm{Si}$ alloys on both sides of the Co layer to account for the mixing between $\mathrm{Co}$ and $\mathrm{Si}$ at both the $\mathrm{Co} / \mathrm{Si}$ interfaces as shown in Fig. 5.

Results obtained from the analysis of the XRR data are presented in Table 1. The structural details of our system as found from the XRR analysis can be understood in conjunction with the AES results in the following way. XRR analysis for the as-deposited sample shows that the thicknesses of the $\mathrm{Si}$ and the Co layer $\left(\mathrm{d}_{2}\right.$ and $\mathrm{d}_{4}$ in Fig.5) are $4.44 \mathrm{~nm}$ and $12.01 \mathrm{~nm}$ 


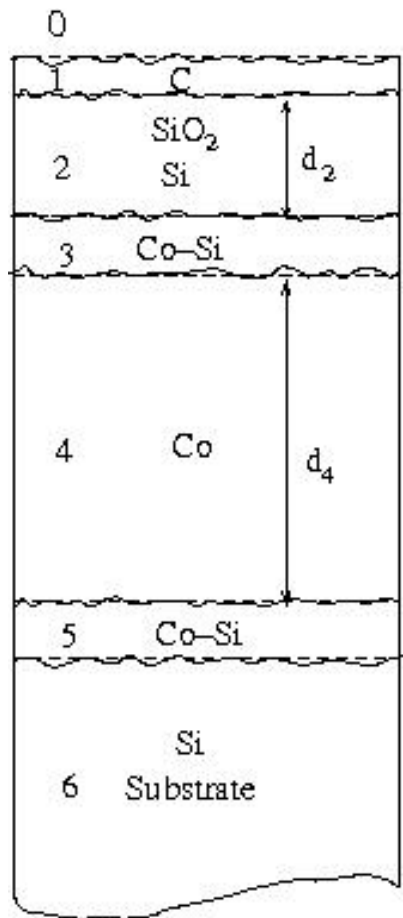

Fig. 5 The pictorial representation of layered structure model which fits best the experimental data. All the fitted parameters are listed in Table I. Two layers (layer 3 and 5) of $\mathrm{Co}_{x} \mathrm{Si}_{1-x}$ have been considered at the $\mathrm{Co} / \mathrm{Si}$ interfaces. The top layer ' 0 ' is vacuum.
(Table 1) respectively. These values are nearly the same as the nominal thicknesses obtained during deposition. The total thickness of the thin film $\left(\mathrm{d}_{1}+\mathrm{d}_{2}+\mathrm{d}_{3}+\mathrm{d}_{4}+\mathrm{d}_{5}\right)$ as we found out from the XRR analysis is $21 \mathrm{~nm}$. These thicknesses are also in good agreement with AES measurements for the as-deposited sample shown in fig. 3. Also the values of electron densities $\left(\rho_{2}\right.$ and $\left.\rho_{4}\right)$ of the above-mentioned layers as found from the XRR analysis (Table 1) are close to the bulk values for $\mathrm{Si}\left(0.70 \times 10^{3}\right.$ electrons $\left./ \mathrm{nm}^{3}\right)$ and $\mathrm{Co}\left(2.48 \times 10^{3}\right.$ electrons $\left./ \mathrm{nm}^{3}\right)$ respectively. The slightly higher estimated value of $\rho_{4}$ for $\mathrm{Co}$ in the as-deposited sample is within the error bar from the bulk value. Critical angle for specular reflection of $\mathrm{X}$-ray depends upon the electron density of the medium from which $\mathrm{x}$-rays are reflected. The minimum angular step for the rotation of the sample in our experiment was $0.002^{\circ}$. The calculated error in $\rho$ due to this uncertainty in measurement of critical angle is about \pm $2 \%$ and the value of $\rho_{4}$ is in agreement with the bulk value under this error bar. Since electron densities of $\mathrm{Si}$

Table 1 Results of analysis of XRR data from the as-deposited as well as ion beam irradiated samples for different fluences. Interpretation of the symbols: $\rho_{i}$ and $d_{i}$ denotes the electron density and the thickness of the $i$-th layer, $\sigma_{i j}$ stands for the interface roughness between the $i$-th and the $j$-th layer and $\sigma_{0}$ stands for the surface roughness, where $i, j=0,1,2$, etc. (as depicted in Fig. 5). Typical error bars for layer thickness and roughness are less than $0.1 \mathrm{~nm}$.

\begin{tabular}{|c|c|c|c|c|}
\hline Ion-fluence & 0 (As- deposited) & $3 \times 10^{14}$ ions $/ \mathrm{cm}^{2}$ & $6 \times 10^{14}$ ions $/ \mathrm{cm}^{2}$ & $1 \times 10^{15}$ ions $/ \mathrm{cm}^{2}$ \\
\hline$\sigma_{0}(\mathrm{~nm})$ & 0.28 & 0.70 & 0.68 & 0.66 \\
\hline$\rho_{1}\left(10^{3}\right.$ electron $\left./ \mathrm{nm}^{3}\right)$ & 0.22 & 0.47 & 0.45 & 0.56 \\
\hline $\mathrm{d}_{1}(\mathrm{~nm})$ & 1.73 & 1.78 & 1.75 & 1.42 \\
\hline$\sigma_{12}(\mathrm{~nm})$ & 0.27 & 0.02 & 0.28 & 0.41 \\
\hline$\rho_{2}\left(10^{3}\right.$ electrons $\left./ \mathrm{nm}^{3}\right)$ & 0.78 & 1.03 & 1.09 & 1.09 \\
\hline $\mathrm{d}_{2}(\mathrm{~nm})$ & 4.44 & 1.71 & 1.70 & 1.69 \\
\hline$\sigma_{23}(\mathrm{~nm})$ & 0.21 & 0.57 & 0.6 & 0.84 \\
\hline$\rho_{3}\left(10^{3}\right.$ electrons $\left./ \mathrm{nm}^{3}\right)$ & 1.45 & 1.81 & 1.87 & 1.65 \\
\hline $\mathrm{d}_{3}(\mathrm{~nm})$ & 1.44 & 2.45 & 2.55 & 1.96 \\
\hline$\sigma_{34}(\mathrm{~nm})$ & 0.03 & 0.82 & 0.88 & 1.48 \\
\hline$\rho_{4}\left(10^{3}\right.$ electrons $\left./ \mathrm{nm}^{3}\right)$ & 2.53 & 2.39 & 2.38 & 2.29 \\
\hline $\mathrm{d}_{4}(\mathrm{~nm})$ & 12.01 & 9.43 & 9.13 & 9.01 \\
\hline$\sigma_{45}(\mathrm{~nm})$ & 0.55 & 1.12 & 1.23 & 1.40 \\
\hline$\rho_{5}\left(10^{3}\right.$ electrons $\left./ \mathrm{nm}^{3}\right)$ & 1.52 & 1.66 & 1.46 & 1.35 \\
\hline $\mathrm{d}_{5}(\mathrm{~nm})$ & 1.38 & 2.43 & 3.84 & 4.67 \\
\hline$\sigma_{56}(\mathrm{~nm})$ & 0.05 & 0.45 & 0.44 & 0.30 \\
\hline$\rho_{6}\left(10^{3}\right.$ electrons $\left./ \mathrm{nm}^{3}\right)$ & 0.70 & 0.70 & 0.72 & 0.71 \\
\hline
\end{tabular}


and $\mathrm{SiO}_{2}$ are nearly the same, x-rays cannot differentiate between these two appreciably and hence we take them as a single material in the XRR analysis, though its presence is clear from the AES results. The heat of mixing between $\mathrm{Co}$ and $\mathrm{Si}$ is negative $(-12 \mathrm{kcal}$ $\mathrm{g}^{-1}$ atom $^{-1}$ ) which means that it is a highly miscible system in solid solution [24]. Keeping this in mind we take up two thin layers of Co-Si alloy at two interfaces at the top and at the bottom of the Co layer in our model (Fig. 5) and determine the electron-density and the layer thickness of these alloy layers (Table 1). The surface roughness $\left(\sigma_{0}\right)$ increases compared to the as-deposited sample due to ion beam bombardment, as observed from Table 1. On the average the interface roughnesses $\left(\sigma_{\mathrm{ij}}\right)$ increase with increase in ion fluence, since by gaining energies from collisions more number of atoms migrate across the interfaces with increasing ion fluences. XRR analysis also shows that the electron density $\left(\rho_{1}\right)$ of the top $\mathrm{C}$ layer increases considerably whereas its thickness $\left(d_{1}\right)$ increases only slowly with increase in ion fluence. This fact is also very apparent from the AES results. The most important effect of ion-beam irradiation in this case as we see from Table 1 is the increase in thicknesses of the Co-Si mixed layers $\left(d_{3}\right.$ and $\left.d_{5}\right)$ at the expense of the thickness of the Co layer $\left(d_{4}\right)$. These results are also strongly supported by AES depth profile measurements. For $2 \mathrm{MeV} \mathrm{Cu}$ ions, the electronic energy losses in $\mathrm{Co}$ and $\mathrm{Si}$ are 2.28 $\mathrm{keV} / \mathrm{nm}$ and $1.19 \mathrm{keV} / \mathrm{nm}$ respectively, while the corresponding nuclear energy losses (due to elastic collisions) are $0.89 \mathrm{keV} / \mathrm{nm}$ and $0.31 \mathrm{keV} / \mathrm{nm}$ respectively [36]. Electronic energy losses of these magnitudes are too small to cause inter-atomic mixing [37]. However, nuclear energy loss or elastic collision can cause ballistic mixing. Energetic $\mathrm{Cu}$ ions can impart higher momentum to Co atoms, compared to $\mathrm{Si}$ atoms, since $\mathrm{Cu}$ and $\mathrm{Co}$ are of comparable masses. Hence more Co atoms than $\mathrm{Si}$ atoms are displaced due to ion beam bombardment in the system [4]. This may partially be responsible for the Co-Si alloy formation and the increase of $d_{5}$ and $d_{3}$ at the expense of $d_{4}$ with increasing ion fluence. In ion irradiation, alloy formation may also be influenced by chemically driven atomic migration across the interfaces [38, 39]. Alloy formation by mixing of $\mathrm{Co}$ and $\mathrm{Si}$ at the interfaces is evident and the mixing increases with increase in ion-beam fluence. Incorporation of some $\mathrm{Si}$ into the cobalt layer reduces the electron density of the Co layer $\left(\rho_{4}\right.$ in Table 1$)$.

\subsection{Exchange Bias and Its Ion Beam Induced Evolution}

Now let us look for the possible interpretation(s) for the observed magnetic exchange bias and its enhancement with increase in ion-fluence in our system. The presence of $H_{E}$ in the as-grown sample may be attributed to the presence of a very small stray magnetic field during the deposition of the Co film. It is also known that such a magnetic field when present during the growth of FM/AFM system shows exchange bias [40] since growth or synthesis of AFM layer in presence of such magnetic field can be, in some cases looked upon as equivalent to 'field cooling'(FC) [16]. However, EB properties remains present if the AFM-FM system is zero field-cooled from a remanent state [13]. In our as-deposited trilayer sample the $\mathrm{M}-\mathrm{H}$ loop (taken at $30 \mathrm{~K}$, not shown here) for FC and $\mathrm{ZFC}$ almost superimpose on each other. The EB effect is still observed in both ZFC and FC M-H loop measurements. Values of $H_{E}$ and $\mathrm{H}_{\mathrm{C}}$ are $3.5 \mathrm{Oe}$ and $12.4 \mathrm{Oe}$ respectively, as obtained from $\mathrm{M}-\mathrm{H}$ plots corresponding to both FC and ZFC (data not presented here). The small stray magnetic field which might have been present during the growth was enough to make this FM $(\mathrm{Co})$ layer saturated at RT, bulk $\mathrm{T}_{\mathrm{C}}$ of Co being much greater than RT [41]. That is why every M-H loop starts with a positive $\mathrm{M}$ even when there is no applied filed $(\mathrm{H}=0)$ in the SQUID measurements. We argue that the two Co-Si alloy layers formed at the interfaces plays the role of 'pinning' layers in this case. An earlier study on multilayers made of alternating layers of $\mathrm{Co}_{x} \mathrm{Si}_{1-\mathrm{x}}$ alloy and $\mathrm{Si}$, for $x \sim 0.75$ showed AFM coupling even at RT [27]. The presence of such 
$\mathrm{Co}_{x} \mathrm{Si}_{1-\mathrm{x}}$ domains in the Co-Si mixed layers at the interfaces may be thought of constructing a multilayer (though not periodic in that sense) of $\mathrm{Co}_{x} \mathrm{Si}_{1-\mathrm{x}}$ and $\mathrm{Si}$. This AFM system magnetically coupled with FM Co layer might be responsible for the EB effect even at RT. We believe there is an antiferromagnetic spin coupling at the AFM/FM interfaces which possibly leads to the positive $\mathrm{EB}$ (i.e. $H_{E}>0$ ) at $\mathrm{RT}$ and lower temperatures. On the nature of the $\mathrm{Co}_{x} \mathrm{Si}_{1-x}$ alloy layers at the interface, we provide further discussions at the end of this section.

We explain the ion irradiation induced enhancement of exchange bias below. Under the macroscopic model invoked by Meiklejohn and Bean $[10,11]$ the phenomenological expression for EB field in a thin film can be written as [16],

$$
H_{E}=J_{E} / M_{S} t_{F M}
$$

where, $J_{E}$ is known as the exchange coupling constant between FM-AFM interface which is essentially equal to half of the change in the interfacial exchange energy $(\Delta \sigma)[40]$ upon a reversal of magnetization of the FM layer; $M_{S}$ and $t_{F M}$ are the saturation magnetization (saturation magnetic moment per unit volume) of the FM layer and the thickness of the FM layer, respectively. Table 2 shows the values of magnetization $\left(M_{S}\right)$ for the as-deposited and the ion irradiated films. For the as-deposited film the magnetization is $\sim 744$ e.m.u. $/ \mathrm{cm}^{3}$. For bulk Co the value of magnetization is $1400 \mathrm{emu} / \mathrm{cm}^{3}$ [41]. For thin films, this value is usually smaller. For Co thin film growth on GaAs, $M_{S}$ values in the range of $800-1000$ e.m.u. $/ \mathrm{cm}^{3}$ have been reported [41]. As the $M_{S}$ value is found to depend on thickness [41], in order to study the ion beam induced effect unambiguously, we ensure that all the samples for irradiation are from the same source as the as-deposited film (cut from a $100 \mathrm{~mm}$ dia sample). Table 2 explicitly shows that with the increase in ion-fluence $t_{F M}\left(\mathrm{~d}_{4}\right)$ decreases. Now increase in $H_{E}$ with reduction in FM layer thickness is quite apparent from Table 2. This fact has been repeatedly reported for almost every thin film EB systems till date and our results are in agreement with them. This variation ensures that EB is purely an interface phenomenon. But unlike some other cases, $H_{E}$ is not inversely proportional to $1 / t_{F M}$ in our case, as the saturation magnetization, $M_{S}$ (Table 2) is not constant but decreases with increase in ion fluence. This reduction is due to some $\mathrm{Si}$ incorporation into the Co layer causing a gradual decrease in the electron density of the Co layer $\left(\rho_{4}\right.$ in Table 1$)$ with increase in ion fluences. In our case $H_{E} \propto 1 / M_{S} t_{F M}$ and this variation (calculated from the parameters listed in Table 2) is observed in Fig. $2 b$ as indicated by the broken line curve. These estimated values reasonably match with the experimental values of $H_{E}$ as measured directly from the M-H loops (Fig. 2a) using Eq. (1), with an estimated value of $J_{E}=0.0036 \mathrm{erg} / \mathrm{cm}^{2}$. This value of exchange coupling constant is in good agreement with those obtained for traditional FM/AFM EB systems [13].

It is also worth mentioning here that a $\mathrm{Co}_{x} \mathrm{Si}_{1-\mathrm{x}}$ alloy can behave as ferromagnetic or nonmagnetic material depending upon the Co concentration $(x)$ in the alloy. The range of $x$ for which a thin film of $\mathrm{Co}_{x} \mathrm{Si}_{1-\mathrm{x}}$ is ferromagnetic is controversial. In some reports thin films of $\mathrm{Co}_{x} \mathrm{Si}_{1-\mathrm{x}}$ are shown to exhibit high coercivity for $x \geq 0.76$ and nonmagnetic for $\mathrm{x}<0.65$ [42], whereas others found them to be ferromagnetic at RT for $x>0.55$ [43]. In our system assuming a homogeneous mixing at the $\mathrm{Co} / \mathrm{Si}$ interfaces we found the Co concentrations $(x)$ at the two Co-Si mixed layers using the simple dilution formula,

$$
\rho_{C o-S i}=x \rho_{C o}+(1-x) \rho_{S i}
$$

where, $\rho_{S i}, \rho_{C o}$ and $\rho_{C o-S i}$ are the electron densities of Si, $\mathrm{Co}$ and the mixed Co-Si layer respectively as obtained from Table 1. The calculated average values of $x$ for the top and the bottom Co-Si layers (figure 5) are 0.42 and 0.45 respectively, in the as-deposited sample and increases gradually to 0.59 and 0.58 respectively in the sample irradiated with the highest ion-fluence. Of course, these values are average concentrations of $\mathrm{Co}$ in the mixed Co-Si layers. There can be a lateral inhomogeneity in the Co concentration. There have been 
Table $2 H_{E}$, as measured directly from the hysteresis loops and $M_{S}$ calculated from the measured saturation magnetic moment are listed along with the FM Co-layer thicknesses $\left(t_{F M}\right)$, which are the $d_{4}$ values obtained from the XRR analysis (Table 1$)$.

\begin{tabular}{llll}
\hline Ion-fluence $\left(\right.$ ions $\left./ \mathrm{cm}^{2}\right)$ & Exchange bias field $H_{E}(\mathrm{Oe})$ & $\begin{array}{l}\text { Saturation magnetic moment/volume } \\
\left(\mathrm{e} . \mathrm{m} . \mathrm{u} . / \mathrm{cm}^{3}\right)\end{array}$ & $\begin{array}{l}M_{S} \text { Ferromagnetic } \\
\text { layer-thickness } t_{F M}(\mathrm{~nm})\end{array}$ \\
\hline 0 (As-deposited) & 3.94 & $7.438 \times 10^{2}$ & 12.01 \\
$3 \times 10^{14}$ & 4.02 & $8.156 \times 10^{2}$ & 9.43 \\
$6 \times 10^{14}$ & 5.03 & $7.886 \times 10^{2}$ & 9.13 \\
$1 \times 10^{15}$ & 7.90 & $5.248 \times 10^{2}$ & 9.01 \\
\hline
\end{tabular}

a few recent studies on the $\mathrm{Co}_{x} \mathrm{Si}_{1-\mathrm{x}} / \mathrm{Si}$ systems [27, 42]. The Curie temperatures of these ferromagnetic $\mathrm{Co}_{x} \mathrm{Si}_{1-\mathrm{x}}$ alloys are above RT [27, 43]. Small local variation in compositions could produce $\mathrm{Si}$ rich non-magnetic regions where surface magnetic poles, and the corresponding AFM dipolar interactions, may appear. AFM coupling in a $\mathrm{Co}_{x} \mathrm{Si}_{1-\mathrm{x}}$ layer has been observed and explained in terms of formation of magnetic/non-magnetic regions as a consequence of local fluctuations of the alloy composition [44]. In our case this local fluctuations in composition is likely to be responsible for the AFM order in the $\mathrm{Co}_{x} \mathrm{Si}_{1-\mathrm{x}}$ layers.

It is to be noted that exchange bias effect can be observed for FM/AFM, FM/FM and FM/spin glass systems. Here the $\mathrm{Co}$ is the FM layer. For the $\mathrm{Co}_{x} \mathrm{Si}_{1-\mathrm{x}}$ alloys no spin glass behaviour has so far been reported. So the alloys have the possibility of being FM or AFM. However Eq. (3) has been found to be followed by FM/AFM systems. This indirectly indicates that the $\mathrm{Co}_{x} \mathrm{Si}_{1-\mathrm{x}}$ alloy layers in our system are AFM. The nature of this interface $\mathrm{Co}_{x} \mathrm{Si}_{1-\mathrm{x}}$ layers need to be further investigated, perhaps by neutron scattering experiments.

A bias shift can also be observed in an exchange spring system with coupled hard and soft magnetic phase. One would expect a large coercive field in such systems. For the present system, the coercive field is about $10 \mathrm{Oe}$, which is too low to have hard FM phase. Often high filed measurements are used to distinguish between exchange bias and exchange spring system. In our case the saturation magnetic moment/volume is 7.4 $\times 10^{2} \mathrm{e} \cdot \mathrm{m} \cdot \mathrm{u} / \mathrm{cm}^{3}$ (Table 2) which is close to the value of saturation magnetic moment of Co thin films [41]. So the high filed measurements are not expected to give any new information. Secondly, The M vs. H hysteresis loop of exchange spring generally shows constricted structure, with a neck like feature around the origin. For the present sample $\mathrm{M}-\mathrm{H}$ loop is square shaped without such anomaly $[45,46]$.

\section{Conclusions}

The authors have carried out magnetic hysteresis loop (M-H loop) measurements on ion beam irradiated and as-grown $\mathrm{Si} / \mathrm{Co}$ bilayers, deposited on a $\mathrm{Si}$ substrate under ultrahigh vacuum condition. Magnetic exchange bias has been observed at room temperature in all the samples and the exchange bias field $H_{E}$ is found to increase with increase in ion-fluence. We speculate that the appearance of $H_{E}$ is due to the presence of antiferromagnetic $\mathrm{Co}_{x} \mathrm{Si}_{1-\mathrm{x}}$ alloy layers at the $\mathrm{Co} / \mathrm{Si}$ interfaces. These layers act as 'pinning' antiferromagnetic layers to the magnetically 'pinned' ferromagnetic Co layer. Depending on the values of $x, \mathrm{Co}_{x} \mathrm{Si}_{1-\mathrm{x}}$ alloys are ferromagnetic (for large values of $x$ ) or non-magnetic (for small values of $x$ ). Formation of magnetic/non-magnetic regions as a consequence of local fluctuations of the alloy composition, is apparently responsible for the antiferromagnetic coupling in the $\mathrm{Co}_{x} \mathrm{Si}_{1-\mathrm{x}}$ alloy layers. The enhancement of $H_{E}$ with ion-fluence is understood from the reduction of the Co layer thickness $\left(t_{F M}\right)$ due to the formation of $\mathrm{Co}_{x} \mathrm{Si}_{1-\mathrm{x}}$ alloys at the interfaces along with some $\mathrm{Si}$ incorporation into the Co layer, which reduces the saturation magnetization $\left(M_{S}\right)$ of the Co layer. The $H_{E} \propto 1 / M_{S} t_{F M}$ behaviour observed here, has also been observed in many FM/AFM systems. From the dependence of $H_{E}$ on the product $M_{S} t_{F M}$ the exchange coupling constant between the ferromagnetic (Co)/ antiferromagnetic 
$\left(\mathrm{Co}_{x} \mathrm{Si}_{1-\mathrm{x}}\right)$ interface has been estimated to be $3.6 \times 10^{-3}$ $\mathrm{erg} / \mathrm{cm}^{2}$. The observed positive values of $H_{E}$ may be attributed to an antiferromagnetic coupling at the interfaces between the ferromagnetic Co layer and the antiferromagnetic $\mathrm{Co}_{x} \mathrm{Si}_{1-\mathrm{x}}$ alloy layers. Observation of $H_{E}$ and its tuning by an accelerated ion-beam at RT gives rise to a possibility of applications of such thin film systems in spin-electronic devices. Therefore, in-depth investigations of their interface spin structure as well as the microstructures are very important. Further investigations on the correlation between these structures and the magnetic behavior are desirable.

\section{Acknowledgments}

SR and BND acknowledge the financial support from the Department of Science and Technology (DST), Government of India for their visit to Hamburg Synchrotron Radiation Laboratory (HASYLAB) at DESY, Germany for carrying out the XRR experiments described in this work. We thank G. Beddies for providing the as-grown sample. We acknowledge the help of the DST unit on Nanoscience at the Indian Association for the Cultivation of Science for SQUID magnetometry. The work has been partly supported under the IBIQuS project.

\section{References}

[1] D. Weller, J.E.E. Baglin, A.J. Kellock, K.A. Hannibal, M.F. Toney, G. Kusinski, S. Lang, L. Folks, M.E. Best, B.D. Terris, Ion induced magnetization reorientation in $\mathrm{Co} / \mathrm{Pt}$ multilayers for patterned media, J. Appl. Phys. 87 (2000) 5768-5770.

[2] S. Bera, K. Bhattacharjee, G. Kuri, B.N. Dev, Probing atomic migration in nanostructured multilayers: Application of X-Ray standing wave fields, Phys. Rev. Lett. 98 (2007) 196103.

[3] B.N. Dev, S. Bera, B. Satpati, D.K. Goswami, K. Bhattacharjee, P.V. Satyam, K. Yamashita, O.M. Liedke, K. Potzger, J. Fassbender, F. Eichhorn, R. Groetzschel, Nonmagnetic to magnetic nanostructures via ion irradiation, Microelectron. Eng. 83 (2006) 1721-1725.

[4] S.K. Ghose, D.K. Goswami, B. Rout, B.N. Dev, G. Kuri, G. Materlik, Ion-irradiation-induced mixing, interface broadening and period dilation in $\mathrm{Pt} / \mathrm{C}$ multilayers, Appl. Phys. Lett. 79 (2001) 467-469.

[5] M.N. Baibich, J.M. Broto, A. Fert, F.N.V. Dau, F. Petroff, P. Etienne, G. Creuzet, A. Friederich, J. Chazelas, Giant Magnetoresistance of $(001) \mathrm{Fe} /(001) \mathrm{Cr}$ magnetic superlattices, Phys. Rev. Lett. 61 (1998) 2472-2475.

[6] G. Binasch, P. Grünberg, F. Saurenbach, W. Zinn, Enhanced magnetoresistance in layered magnetic structures with antiferromagnetic interlayer exchange, Phys. Rev. B 39 (1989) 4828-4830.

[7] S.S.P. Parkin, C. Chappert, F. Hermann, Oscillatory exchange coupling and giant magnetoresistance via $\mathrm{Cu}-\mathrm{X}$ Alloys (X = Au, Fe, Ni), Europhys. Lett. 24 (1993) 71-76.

[8] S. Mathew, B. Satpati, B. Joseph, B.N. Dev, R. Nirmala, S.K. Malik, R. Kesavamoorthy, Magnetism in $\mathrm{C}_{60}$ films induced by proton irradiation, Phys. Rev. B 75 (2007) 075426.

[9] J. Fassbender, D. Ravelosona, Y. Samson, Tailoring magnetism by light ion-irradiation, J. Phys. D: Appl. Phys. 37 (2004) R179-R196.

[10] W.H. Meiklejoh, C.P. Bean, New magnetic anisotropy, Phys. Rev. 102 (1956) 1413-1414.

[11] W.H. Meiklejohn, C.P. Bean, New magnetic anisotropy, Phys. Rev. 105 (1957) 904-913.

[12] M. Ali, P. Adie, C.H. Marrows, D. Greig, B.J. Hickey, R.L. Stamps, Exchange bias using a spin glass, Nature Mater 6 (2007) 70-73.

[13] J. Nogués, I.K. Schuller, Exchange bias, J. Magn. Magn. Mater.192 (1999) 203-232.

[14] S.G.E. Velthuis, G.P. Felcher, J.S. Jiang, A. Inomata, C.S. Nelson, A. Berger, S.D. Bader, Magnetic configurations in exchange-biased double superlattices, Appl. Phys. Lett. 75 (1999) 4174-4176.

[15] F. Canet, A. Mangin, C. Bellouard, M. Piecuch, Positive exchange bias in ferromagnetic-ferrimagnetic bilayers: FeSn/FeGd, Europhys. Lett. 52 (2000) 594-600.

[16] J. Nogués, J. Sort, V. Langlais, V. Skumryev, S. Surinach, J.S. Munoz, M.D. Baró, Exchange bias in nanostructures, Phys. Rep. 422 (2005) 65-117.

[17] Y. Shimada, H. Kojima, Magnetic properties of amorphous Fe-Si thin films, J. Appl. Phys. 47 (1976) 4156-4159.

[18] L.M. Alvarez-Prado, G.T. Peréz, R. Morales, F.H. Salas, J.M. Alameda, Perpendicular anisotropy detected by transversely biased initial susceptibility via the magneto-optic Kerr effect in $\mathrm{Fe}_{x} \mathrm{Si}_{1-x}$ thin films and $\mathrm{Fe}_{x} \mathrm{Si}_{1-x} / \mathrm{Si}$ multilayers: Theory and experiment, Phys. Rev. B 56 (1997) 3306-3316.

[19] J.M. Alameda, J.M. González, F. Lopéz, J.L. Vincent, Magnetic properties of amorphous sputtered $\mathrm{Co}_{\mathrm{x}} \mathrm{Si}_{1-\mathrm{x}}$ films, J. Magn. Magn. Mater. 38 (1983) 105-108. 
[20] J.M. Fallon, C.A. Faunce, P.J. Grundy, The structure and properties of sputter-deposited Co-Si alloy thin films, J. Phys. Condens. Matter. 12 (2000) 4075-4089.

[21] S. Toscano, B. Briner, H. Hopster, M. Landolt, Exchange-coupling between ferromagnets through a non-metallic amorphous spacer-layer, J. Magn. Magm. mater. 114 (1992) L6-L10.

[22] E.E. Fullerton, J.E. Mattson, S.R. Lee, C.H. Sowers, Y.Y. Huang, G. Felcher, S.D. Bader, Non-oscillatory antiferromagnetic coupling in sputtered $\mathrm{Fe} / \mathrm{Si}$ superlattices, J. Magn. Magn. Mater. 117 (1992) L301-L306.

[23] J.M. Pruneda, R. Robles, S. Bouarab, J. Ferrer, A. Vega, Antiferromagnetic interlayer coupling in $\mathrm{Fe} / \mathrm{c}-\mathrm{SiFe} / \mathrm{Fe}$ sandwiches and multilayers, Phys. Rev. B 65 (2001) 024440 .

[24] I. Sakamoto, S. Honda, H. Tanoue, M. Koike, S. Purwanto, Structural and magnetic properties of $\mathrm{Fe} / \mathrm{Si}$ and $\mathrm{Fe} / \mathrm{FeSi}$ multilayers, J. Magn. Magn. Mater. 290-291 (2005) 78-81.

[25] I.M. Fallon, C.A. Faunce, P.J. Grundy, Ion beam modified Co/Si multilayers, J. Appl. Phys. 87 (2000) 6833-6835.

[26] J M. Fallon, C.A. Faunce, P.J. Grundy, Microstructure of sputter-deposited Co/Si multilayer thin films, J.Appl. Phys. 88 (2000) 2400-2407.

[27] C. Quirós, J.I. Martin, L. Zárate, M. Vélez, J. Alameda, Antiferromagnetic coupling in amorphous $\mathrm{Co}_{\mathrm{x}} \mathrm{Si}_{1-x} \mathrm{Si}$ multilayers, Phys. Rev. B 71 (2005) 024423.

[28] T. Mewes, R. Lopusnik, J. Fassbender, B. Hillebrands, M. Jung, D. Engel, A. Ehresmann, H. Schmoranger, Suppression of exchange bias by ion irradiation, Appl. Phys. Lett. 76 (2000) 1057-1059.

[29] A. Mougin, T. Mewes, R. Lopusnik, M. Jung, D. Engel, A. Ehresmann, H. Schmoranger, J. Fassbender, B. Hillebrands, Modification of the exchange bias effect by He ion irradiation, IEEE Trans. Magn. 36 (2000) 2647.

[30] J.K. Tripathi, P.C. Srivastava, Ion irradiation induced nano granular magnetic $\mathrm{Fe}_{5} \mathrm{Si}_{3}$ silicide phase formation in $\mathrm{Fe} / \mathrm{Si}$ structures, Appl. Surf. Sci. 255 (2008) 2767-2772.

[31] U. Pietsch,V. Holý, T. Baumbach, High-resolution X-ray Scattering from Thin Films to Lateral Nanostructures, 2nd Ed., Springer, 2004.

[32] J. Daillant, A. Gibaud, X-ray and Neutron Reflectivity: principles and applications, Springer-Verlag, Berlin, 1999.

[33] S. Vitta, Structural investigation of thin films and multilayers using X-ray scattering, Curr. Sci. 79 (2000) 61-69.

[34] S.K. Ghos, B.N. Dev, X-ray standing wave and reflectometric characterization of multilayer structures,
Phys. Rev. B 63 (2001) 245409 (1-11) and references therein.

[35] S.K. Ghose, Ph.D. thesis, Utkal University, 2001.

[36] J.F. Ziegler, M.D. Ziegler, J.P. Biersack, SRIM-2008.3, available online at: http://www.srim.org.

[37] Z.G. Wang, C. Dufour, E. Paumier, M. Toulemonde, The $\mathrm{S}_{\mathrm{e}}$ sensitivity of metals under swift-heavy-ion irradiation: a transient thermal process, J. Phys.: Condens. Matter 6 (1994) 6733-6750.

[38] S. Bera, B. Satpati, D.K. Goswami, K. Bhattacharjee, P.V. Satyam, B.N. Dev, Ion-beam induced transformations in nanoscale multilayers: evolution of clusters with preferred length scales, J. Appl. Phys. 99 (2006) 074301(1-5).

[39] A. Barna, L. Kotis, J. Lábár, Z. Osváth, A.L. Tóth, M. Menyhárd, A. Zalar, P. Panjan, Producing metastable nanophase with sharp interface by means of focused ion beam irradiation, J. Appl. Phys. 105 (2009) 044305(1-5).

[40] R. Jungblut, R. Coehoorn, M.T. Johnson, J. aan de Stegge, A. Reinders, Orientational dependence of the exchange biasing in molecular-beam-epitaxy-grown $\mathrm{Ni}_{80} \mathrm{Fe}_{20} / \mathrm{Fe}_{50} \mathrm{Mn}_{50}$ bilayers, J. Appl. Phys. 75 (1994) 6659-6664.

[41] Z. Ding, P.M. Thibado, C. Awo-Affouda, V.P. Labella, Electron-beam evaporated cobalt films on molecular beam epitaxy prepared GaAs (001), J. Vac. Sci. Technol. B 22 (2004) 2068-2072.

[42] M. Vélez, S.M. Valvidares, J. Diaz, R. Morales, J.M. Alameda, Structure and magnetic properties of amorphous Co-Si alloy films, IEEE Trans. on Magn. 38 (2002) 3078-3080.

[43] C.N. Afonso, S. Girón, A.R. Lagunas, J.L. Vincent, Transport properties and magneto-optic Kerr effect in sputtered $\mathrm{Co}_{\mathrm{x}} \mathrm{Si}_{1-\mathrm{x}}$ films, IEEE Trans. on Magn. MAG-17 (1981) 2849-2851.

[44] C. Quirós, L. Peverini, L. Zárate, A. Alija, M. Vélez, G. Rodríguez-Rodríguez, F. Fauth, E. Ziegler, J.M. Alameda, Enhancement of antiferromagnetic coupling in magnetic multilayers by low energy ion beam substrate nanopatterning, J. Phys.:Condens. Matter 21 (2009) 224024(1-7).

[45] A. Bill, H.B. Braun, Magnetic properties of exchange springs, J. Mag. Mag. Mater. $272-276$ (2004) 1266-1267.

[46] E.E. Fullerton, J.S. Jiang, M. Grimsditch, C.H. Sowers, S.D. Bader, Exchange-spring behavior in epitaxial hard/soft magnetic bilayers, Phys. Rev. B 58 (1998) 12193-12200. 